Section 2.

IMPROVED DEFINTIONS AND MODELS 


\title{
Definition of the Celestial Ephemeris Pole and the Celestial Ephemeris Origin
}

\author{
Nicole Capitaine \\ DANOF/UMR8630-CNRS, Observatoire de Paris \\ 61, avenue de l'Observatoire, 75014 - Paris, France, \\ capitain@danof.obspm.fr
}

\begin{abstract}
The adoption of the International Celestial Reference System (ICRS) by the IAU in use since 1 January 1998, and the accuracy achieved by the most recent models and observations of Earth rotation call for a redefinition of the Earth Orientation Parameters (EOP). First, the precession-nutation parameters and Greenwich sidereal time, which are currently defined in the FK5 System, have to be re-defined to be consistent with the ICRS. Second, the current definition of the Celestial Ephemeris Pole (CEP) has to be extended in order to be consistent with the most recent models for nutation and polar motion at a microarsecond accuracy including diurnal and sub-diurnal components, as well as with new strategies of observations. Such issues have been under consideration by the subgroup T5 named "Computational Consequences" of the IAU Working Group "ICRS". This paper gives, as the basis for future recommendations, the preliminary proposals of the subgroup T5 for a modern definition of the CEP, for the definition of more basic EOP in the ICRS and for the choice of a new origin on the equator of the CEP in place of the equinox. Then, the paper emphasizes the use of the Celestial Ephemeris Origin (CEO) which is defined as the "non-rotating origin" in the celestial frame on the equator of the CEP.
\end{abstract}

\section{Introduction}

There have been important changes in the situation of Earth rotation during the last 20 years. First, there have been large improvements in the observations since 1980 both in precision and time resolution and in strategy and procedures. An important change is that the celestial pole offsets are estimated on a regular basis by VLBI since 1980 providing the actual position of the pole in the celestial frame.

Second, the International Astronomical Union (IAU) adopted the International Celestial Reference System (ICRS) in use since 1 January 1998 (Ma et al., 1998) such that the barycentric directions of distant extragalactic objects show no global rotation with respect to these objects. The geocentric celestial system used for the transformation between the celestial and the terrestrial system, designated here by CRS, is such that its orientation follows the kinematical condition of absence of global rotation of geocentric directions of the 
objects that realize the ICRS. This provides a non-rotating geocentric celestial frame to measure Earth rotation that has no global rotation and is no longer dependent on the Earth's motion as was previously the case with the FK5.

Third, there have been large improvements in theory at $\mu$ as accuracy, during the period 1990-1999. The theory now includes diurnal and sub-diurnal nutations in the CRS as well as diurnal and sub-diurnal variations in polar motion.

The consequences of this new situation are such that the current parameters for precession, nutation and the Earth's angle of rotation, which are referred to the FK5, are not adapted to the ICRS and that the current definition of the CEP is not consistent with the precision and spatial resolution of modern techniques, nor with the accuracy of theories and frequencies of the included terms. This new situation requires that more basic parameters for precession, nutation and the Earth's angle of rotation be defined with respect to the CRS and that an extended definition of the CEP be given.

Such consequences are under study in the sub-group T5 "Computational Consequences" of the IAU/WG ICRS whose membership is: N. Capitaine, V. Dehant, A.-M. Gontier, B. Kołaczek, J. Kovalevsky, J. Lieske, C. Ma, D.D. McCarthy, O. Sovers, and J. Vondrák. There have been four T5 Newsletters from 1998 to 2000, devoted to discussions of the extended definition of the CEP and of the definition of new parameters for Earth orientation. Other contributors to the discussions of these newsletters have been: C. Bizouard, P. Bretagnon, A. Brzezi/'nski, B. Guinot, S. Loyer, P. Mathews, J. Mueller, L. Petrov, M. Rothacher, H. Schuh, and Y. Yatskiv.

This paper gives the preliminary proposals of the subgroup T5 that are considered as the basis for future recommendations. The proposal for a modern definition of the CEP is discussed in Section 2 and the definition of more basic EOP in the CRS in Section 3 together with the choice of a new origin on the equator of the CEP. Then, the paper emphasizes, in Section 4, the use of the Celestial Ephemeris Origin (CEO) which is defined as the "non-rotating origin" in the CRS on the equator of the CEP, for the definition of Earth rotation and UT1.

\section{Towards a modern definition of the CEP}

\subsection{Current realization of the pole}

The observations of the Earth's orientation are sensitive to the position of the pole of the Terrestrial Reference System (TRS) with respect to the CRS. However, an intermediate pole is considered in the coordinate transformation between the TRS and the CRS. This is currently the Celestial Ephemeris Pole (CEP) as defined in the IAU 1980 theory of nutation (Seidelmann, 1982). The current Earth's angle of rotation as used for estimating UT1 is about the axis of the CEP.

The CEP is an intermediary pole separating, by convention, the motion of the pole of the TRS (i.e. the pole of the mean surface geographic figure axis, or Tisserand mean axis of the Earth) into two parts: 
- a "celestial part", designated as the celestial motion of the CEP, including all the long-period terms in the CRS (precession/nutation) with periods greater than 2 days (i.e. frequencies between $-1 / 2 \mathrm{cpd}$ and $+1 / 2 \mathrm{cpd}$ ),

- a "terrestrial part", designated as the terrestrial motion of the CEP, including all the long-period terms in the TRS (polar motion) with periods greater than 2 days, (i.e. frequencies between $-1 / 2 \mathrm{cpd}$ and $+1 / 2 \mathrm{cpd}$ ).

Such motions can be viewed either in the CRS or in the TRS according to the chosen convention as shown in the schematic representation below.

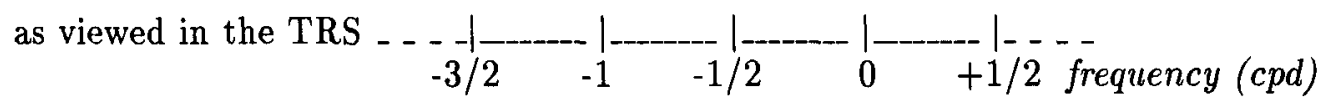

Celestial motion | Terrestrial motion

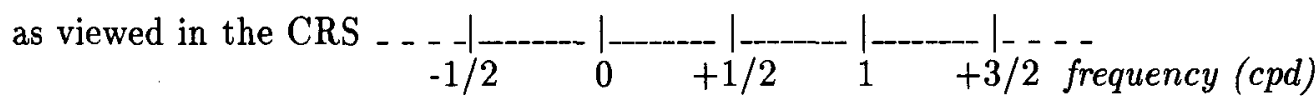

It must be noted that there is no convention for terms outside the frequency interval $[-3 / 2,+1 / 2]$ as viewed in the TRS or $[-1 / 2,+3 / 2]$ as viewed in the CRS. It means that the current definition of the CEP is valid only in this frequency interval and does not include any convention for the high-frequency motions in both the CRS and the TRS. Moreover, the current convention is only valid for sampling intervals greater than 1 day.

\subsection{The need for an extended definition of the CEP}

The main changes for the definition of the CEP (see Capitaine \& Brzezinski, 1999 or Capitaine, 1999 for more details) concern the predictable high-frequency components in nutation (Bretagnon et al., 1997) and in polar motion (Herring \& Dong, 1994) and the new strategy of observations in which sub-daily observations will increase in the near future. The recent models, at a microarsecond accuracy, include:

- prograde semidiurnal nutations with amplitudes of the order of $15 \mathrm{mi}$ croarseconds, produced by the lunisolar torque exerted on the tesseral coefficients $(2,2)$ and $(3,2)$ of the Earth's gravitational potential,

- prograde diurnal nutations (in $\Delta \psi \sin \varepsilon_{0}$ and $\Delta \varepsilon$ ) with amplitudes of the order of 15 microarseconds, due to the tesseral coefficients $(3,1)$ and $(4,1)$,

- prograde diurnal tidal variations in polar motion with amplitudes of the order of 150 microarseconds,

- prograde semidiurnal tidal variations in polar motion with amplitudes of the order of 100 microarseconds,

- retrograde diurnal tidal variations in polar motion with amplitudes of the order of 800 microarseconds, 
- retrograde semidiurnal tidal variations in polar motion with amplitudes of the order of 300 microarseconds.

The overlapping between these high frequency motions is such that:

- the prograde diurnal nutations in space appear in the Earth as long periodic prograde and retrograde variations in polar motion of periods \pm 27 d, $193.6 \mathrm{~d}, 3193.9 \mathrm{~d}, \ldots$ (Bizouard et al., 1999a),

- the semidiurnal prograde nutations in space appear as prograde diurnal variations in polar motion, also called "polar libration" (Chao et al., 1991),

- the retrograde diurnal terms in the tidal polar motion variations are in fact included in the most recent models of nutation for a nonrigid Earth.

The extended definition of the CEP must therefore clearly specify which part of these high frequency components is considered in the CRS or in the TRS on the basis of clear theoretical considerations. For sub-daily observations, the two frequency intervals of polar motion and nutation are no longer separated and thus, the frequency domain decomposition into polar motion and nutation becomes strongly dependent on the method used for processing the observations (Brzeziński \& Capitaine, 1993). Hence, simultaneous estimation of polar motion and nutation must be avoided.

\subsection{Proposal for a modern definition of the CEP}

The discussions in $\mathrm{T} 5$ have led to the following proposals concerning the concept and realization of the CEP:

\section{Concept}

- to abandon the IAU 1980 definition "no nearly-diurnal motion...",

- the pole must not be defined by its realization but a clear concept not dependent on further improvements in the model,

- the pole must be defined such that it can be realized by a model as accurately as necessary,

- the change from the current CEP should have minimal impact on users,

- to consider a change of name and call the new pole "Celestial Reference Pole" (CRP), or "Celestial Intermediate Pole" (CIP),

- to specify the motion in the CRS by a model,

- to sharpen the definition of the CEP by taking into account the high frequency variations of polar motion.

A tentative definition is the Pole of the intermediate equator (between the TRS and $C R S$ ) the motion of which, in the CRS, is produced by the external gravitational forces acting on the Earth after filtering out terms with periods less than 2 days. 


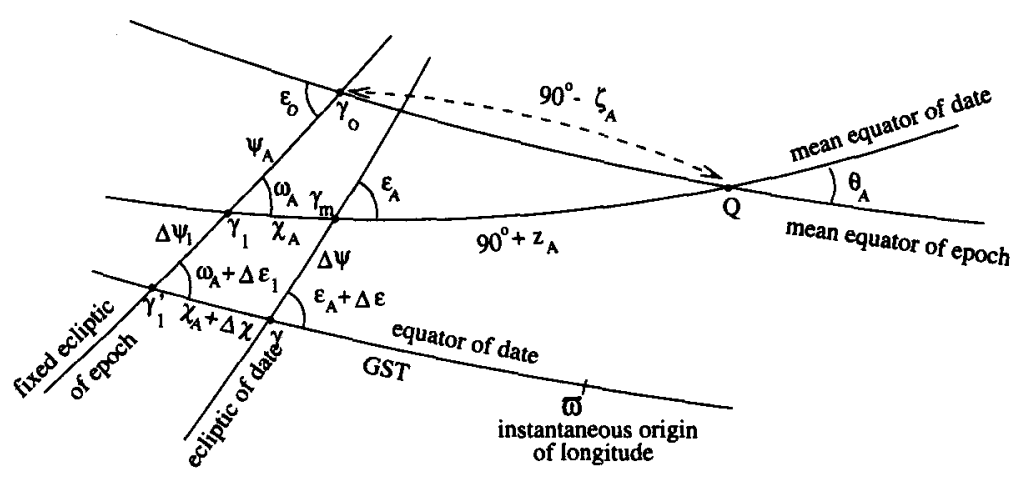

Figure 1. Precession-nutation and Earth's rotation parameters referred to the FK5.

The forced motion of the CEP in the CRS is that of the Tisserand mean axis of the Earth. The nutations with periods less than 2 days (prograde diurnal and prograde semidiurnal nutations) are included in the model for the variations in polar motion.

\section{Realization}

- the motion of the CEP in the CRS is specified by the best available precession-nutation series plus the time dependent celestial pole offsets,

- the motion of the CEP in the TRS is provided by observations taking into account a predictable part specified by an empirical model,

- the corrections to the empirical model can be obtained by extracting the high frequency signal from the pole coordinates either together with the long periodic motion (following the procedure proposed by Mathews (1999) and successfully tested by Bizouard et al. (1999b)), or in a second step.

\section{The Earth Orientation Parameters}

\subsection{The current EOP}

The FK5, based on positions and proper motions of bright stars, was oriented so that at the "epoch", the positions are referred to the best estimate of the location of the mean pole and mean equinox. The proper motions of stars were evaluated so that, for the adopted model of precession, they provide the best access to the mean pole and mean equinox at any other date.

The current precession angles (see Fig. 1) are those defined by Lieske et al. (1977) in the FK5 system. The current nutation angles $\Delta \psi$ in longitude and $\Delta \epsilon$ in obliquity are referred to the ecliptic of date (see Fig. 1) and $\chi_{A}+\Delta \chi$ is the angular distance between the ecliptic of epoch and the ecliptic of date along the equator of date.

The current procedure to account for precession and nutation is to use the matrix transformation $\mathrm{P}(\mathrm{t})$ using the developments as function of time of the precession angles, $\zeta_{A}, \theta_{A}, z_{A}$, followed by the matrix transformation $\mathrm{N}(\mathrm{t})$ using 
the nutation angles $\Delta \psi$ and $\Delta \epsilon$ provided by the conventional series of nutation (Seidelmann, 1982; McCarthy, 1996). Such a transformation corresponds to a sequence of six consecutive rotations for precession and nutation using five different parameters. As the precession and nutation angles are referred to the ecliptic of date, the $\mathrm{PN}(\mathrm{t})$ matrix is dependent both on the precession and nutation of the equator and to the precession of the ecliptic.

The current procedure to account for Earth rotation in the FK5 system is to use the relationship between Greenwich sidereal time and UT1 (Aoki et al., 1982) giving GMST at date $t$, followed by the relationship between GST and GMST and then to take into account the interval of GMST from 0h UT1 to the hour of observation. The difference GST-GMST is provided, since 1 January 1997, by the "complete" equation of the equinoxes (Aoki \& Kinoshita, 1983; McCarthy, 1996). In addition to Earth rotation, the angle GST thus includes (Capitaine \& Gontier, 1993) a part due to the accumulated precession and nutation along the equator as well as a part (in GST-GMST) due to crossed terms between precession and nutation and crossed nutation terms. It refers to the ecliptic of date and thus mixes Earth rotation and precession-nutation. It must also be noted that the secular term in the relationship between GMST and UT1 mixes UT1 and TCG.

\subsection{Earth Orientation Parameters referred to the CRS}

The fundamental properties of the CRS are the absence of global rotation and the abandonment of the link with the motion of the Earth. In this spirit, the definition of the EOP must be based on location and motion of the rotation pole only, without any relation to the orbital motion of the Earth. It is thus necessary to abandon the current parameters in the FK5 System which combine the motions of the equator and the ecliptic with respect to the ICRS. The requirements for the new EOP are the following:

- to be based on clear concepts referred to the ICRS,

- to use a minimal number of parameters,

- not to be referred to the ecliptic of date, but to a fixed plane,

- to abandon the use of the true equinox as the origin on the moving equator,

- to include both precession and nutation of the equator.

Several options can been considered among which the most basic ones, the Euler angles between the CRS and the TRS and the celestial and terrestrial coordinates of the CEP, are described below.

(i) Euler angles between the CRS and the TRS

The two first Euler angles, $\theta$ and $\psi$, include both polar motion and precessionnutation referred to the ecliptic of epoch and the third one, $\phi$, the origin of which is the node of the equator of figure on the ecliptic of epoch, includes both Earth rotation and the precession-nutation motion of the equator of figure. No intermediate pole is used in the transformation between the TRS and the CRS using Euler angles which is based on only three parameters. 


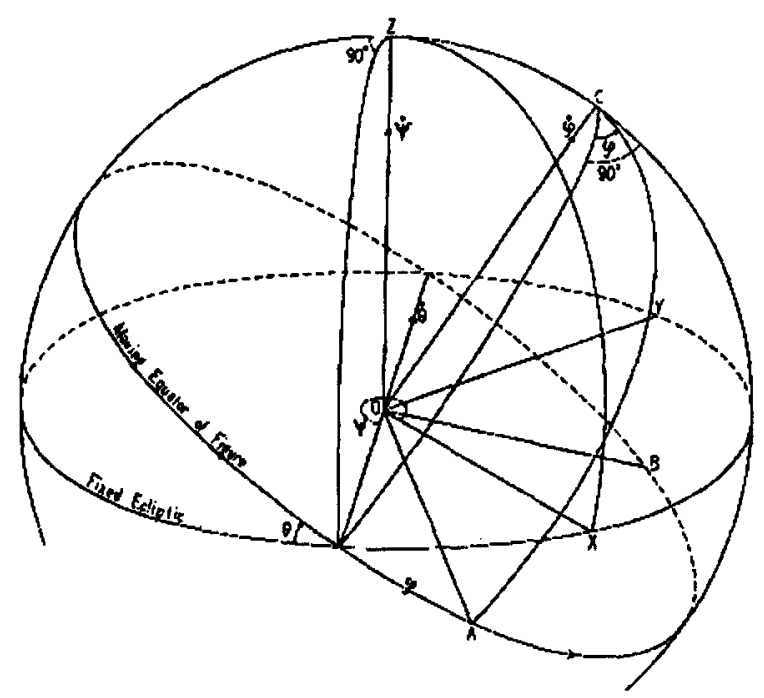

Figure 2. The Euler angles between the terrestrial frame (OABC) and the celestial ecliptic frame (OXYZ) (Woolard, 1953).

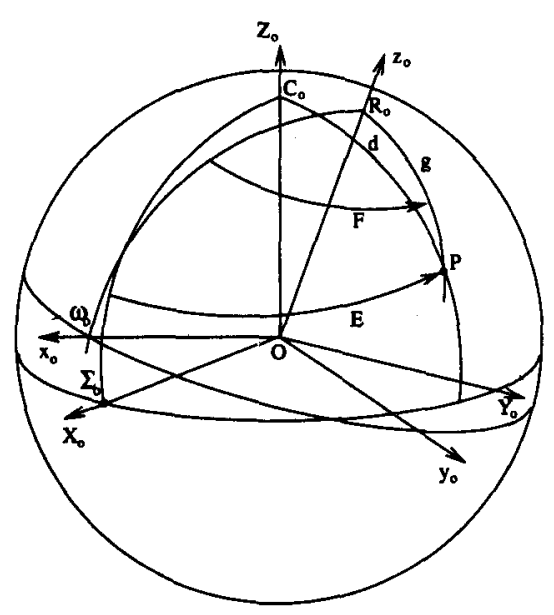

Figure 3. The coordinates of the CEP (noted P) in the CRS and TRS. 


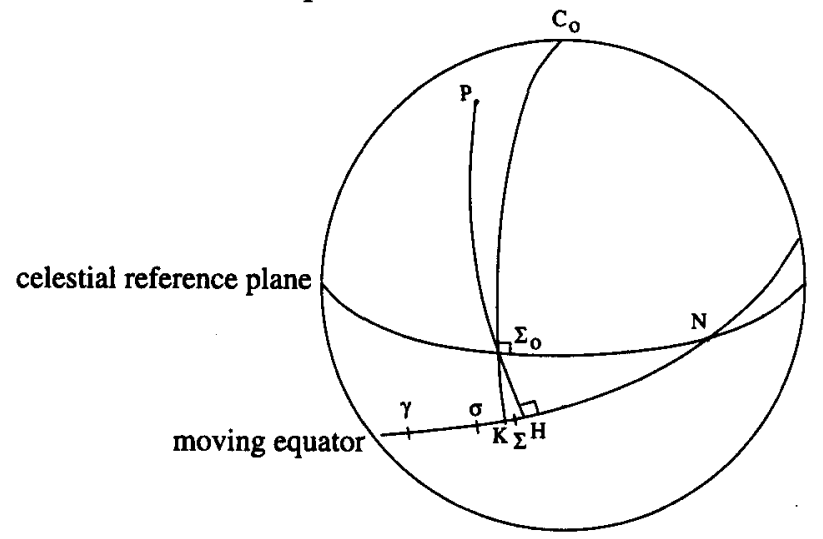

Figure 4. Alternative origins on the moving equator.

(ii) The coordinates of the $C E P$

The coordinates $X=\sin d \cos E, Y=\sin d \sin E$ of the CEP in the CRS (Fig. 3 ) include precession and nutation, the coupling effects between precession and nutation, as well as the offsets $\xi_{o}, \eta_{o}$, between the CEP at J2000.0 and the pole of the CRS and $d \alpha_{0}$ between the mean equinox at J2000.0 and the origin on the equator of the CRS. $X$ and $Y$ can be expressed as (Capitaine, 1990):

$$
\begin{aligned}
X= & \bar{X}+\xi_{0}-d \alpha_{0} \bar{Y}, Y=\bar{Y}+\eta_{0}+d \alpha_{0} \bar{X}, \text { with (see Fig. 1) } \\
& \bar{X}=\sin \left(\omega_{A}+\Delta \omega_{1}\right) \sin \left(\psi_{A}+\Delta \psi_{1}\right) \\
& \bar{Y}=-\sin \epsilon_{0} \cos \left(\omega_{A}+\Delta \omega_{1}\right)+\cos \epsilon_{0} \sin \left(\omega_{A}+\Delta \omega_{1}\right) \cos \left(\psi_{A}+\Delta \psi_{1}\right),
\end{aligned}
$$

the quantities $\psi_{A}+\Delta \psi_{1}$ and $\omega_{A}+\Delta \omega_{1}$ being referred to the ecliptic of epoch.

The celestial pole coordinates are supposed to be referred to an intermediate pole, such as the CEP, and appear in a symmetric form as the coordinates in the TRS, $u=\sin g \cos F, v=\sin g \sin F$.

(iii) The choice of an origin on the moving equator The possible origins on the moving equator to be used in place of the equinox $\gamma$ (intersection with the moving ecliptic), are (see Fig. 4 and Fig. 5):

$\gamma_{1}^{\prime}:$ the intersection with the fixed ecliptic,

$\mathrm{K}:$ the intersection with the zero-line meridian of the CRS,

$\mathrm{H}$ : the intersection with the instantaneous prime meridian $P \Sigma_{O}$,

$\Sigma:$ such that $: \Sigma N=\Sigma_{0} N$,

$\mathrm{N}$ : the node upon the celestial reference great circle,

$\sigma:$ the non rotating origin (Guinot, 1979), such that $\dot{s}=\dot{E}(\cos d-1)$.

A comparison of the parameters considered above shows that the Euler angles, or equivalently the celestial coordinates of the $z$-axis of the TRS, which reduce to three the number of EOP, do not use any intermediate pole. The Euler angles thus include both high-frequency and low-frequency components of the motion of the z-axis of the TRS in the CRS, and their estimation from observations would need a large number of parameters. 


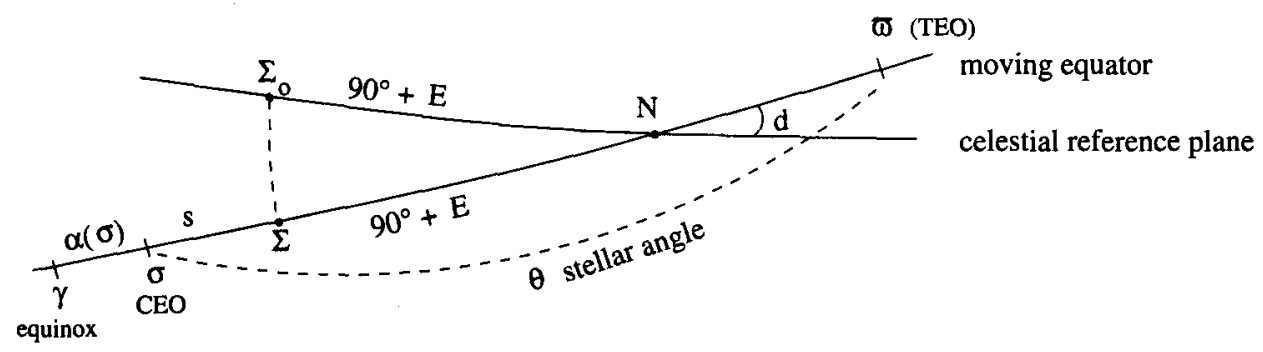

Figure 5. Definition of the CEO, $\sigma$, the TEO, $\varpi$ and the stellar angle $\theta$.

On the contrary, the celestial coordinates of the CEP separate the celestial components from the terrestrial components according to a "frequency criterion" using an intermediate pole. Such a procedure facilitates the estimation of the parameters from observations.

A comparison of the origins considered above, either of geometrical meaning (such as $\Sigma$ or $K$ ) or of kinematical origin $(\sigma)$ shows that, in the first case, the instantaneous rotation of the origin considered is included in the derived value for the Earth's angular rotation, whereas it is clearly separated when using directly the non-rotating origin (NRO) as the origin on the moving equator. The preferred parameters resulting from the discussions of $\mathrm{T} 5$ are the celestial pole coordinates $\mathrm{X}, \mathrm{Y}$, and the preferred origin is the NRO. Section 4 emphasizes the practical use of the NRO in the transformation coordinates between the TRS and the CRS.

\section{Definition and use of the celestial and terrestrial ephemeris origins}

\subsection{Definition of the CEO and TEO}

To define an Earth's angle of rotation that includes only the "intrinsic Earth rotation", it is necessary to eliminate the spurious rotation of the origin on the moving equator due to the precession-nutation of the equator. It is thus necessary to use an origin defined by the kinematical property of having "no instantaneous rotation" around the axis of rotation with respect to the CRS. That provides the "non-rotating origin" $\sigma$ (Guinot, 1979), which can be designated as "the Celestial Ephemeris Origin" (CEO) when referred to the axis of the CEP. Similarly the "Terrestrial Ephemeris Origin" (TEO), $\varpi$, designates the terrestrial NRO. The "stellar angle" is defined by $\theta=\varpi \sigma$ (see Fig. 5) and is such that it includes only Earth rotation.

The positioning of the CEO and TEO are provided by the quantities $s$ and $s^{\prime}$ such that, with $\sigma\left(t_{0}\right) \mathrm{N}_{0}=\Sigma_{0} \mathrm{~N}_{0}$ :

$$
\begin{aligned}
& s=\sigma \mathrm{N}-\Sigma_{0} \mathrm{~N}=\int_{t_{0}}^{t}(\cos d-1) \dot{E} d t=-\int_{t_{0}}^{t} \frac{X \dot{Y}-Y \dot{X}}{1+Z} d t, \\
& s^{\prime}=\varpi \mathrm{M}-\Pi_{0} \mathrm{M}=-\int_{t_{0}}^{t} \frac{u \dot{v}-v \dot{u}}{2} d t=\int_{t_{0}}^{t}(\cos g-1) \dot{F} d t .
\end{aligned}
$$




\subsection{The use of the CEO and the TEO}

The coordinate transformation from the TRS to the CRS using the celestial pole coordinates, the CEO and TEO, is composed of three components $W(t), R(t)$ and $P N(t)$, respectively for polar motion, rotation of the Earth and precessionnutation. They can be written as:

$$
\begin{aligned}
& W(t)=R_{3}\left(-s^{\prime}\right) \cdot R_{1}(-v) \cdot R_{2}(u), \\
& R(t)=R_{3}(-\theta) \\
& P N(t)=R_{3}(-E) \cdot R_{2}(-d) \cdot R_{3}(E) \cdot R_{3}(s) .
\end{aligned}
$$

Development as a function of time of $X$ and $Y$ and $s$ with microarsecond accuracy after one century are provided by Capitaine et al. (2000) consistent with the IERS 1996 precession and nutation and the numerical values of the celestial offsets at J2000.0. Such developments include a constant term, a polynomial form of $t$, a sum of periodic terms and a sum of Poisson terms. Using such parameters, the definition of UT1 takes the simple form: UT1-UT1 $1_{0}=k \theta$, where $k$ is a constant selected so that, on the average, the Sun crosses the prime meridian at 12 hours UT1. This shows that the use of the CEO and the TEO provides EOP corresponding to clear concepts referred to the ICRS.

\section{References}

Aoki, S., Kinoshita, H., 1983, Celest. Mech., 29, 335-360.

Aoki, S., Guinot, B., Kaplan, G. H., Kinoshita, H., McCarthy, D. D., Seidelmann, P. K., 1982, Astron. Astrophys., 105, 359-361.

Bizouard, C., Folgeira, M., Souchay, J., 1999a, in the Proceedings of IAU Colloquium 178, 613-617.

Bizouard, C., Capitaine, N., Gontier, A.-M., Mathews, P., 1999b, to be published in the Proceedings of the Journées Systèmes de Référence SpatioTemporels 1999, in press.

Bretagnon, P., Rocher, P., and Simon, J.-L., 1997, Astron. Astrophys., 319, 305-317.

Brzeziński, A., Capitaine, N., 1993, J. Geophys. Res., 98, No. B4, 6667-6675.

Capitaine, N., 1990, Celest. Mech. Dyn. Astr., 48, 127-143.

Capitaine, N., 1999, in the Proceedings of the IAU Colloquium 178, 573-584.

Capitaine, N., Brzeziński, A., 1999, Proceedings of the Journées Systèmes de Référence Spatio-Temporels 1998, N. Capitaine (ed.), Observatoire de Paris, 155-160.

Capitaine, N., Gontier, A.-M., 1993, Astron. Astrophys., 275, 645-650.

Capitaine, N., Guinot, B., McCarthy, D. D., 2000, Astron. Astrophys., 355, 398-405.

Chao, B. F., Dong, D. N., Herring, T. A., 1991, Geophys. Res. Lett., 18, 2007-2010.

Guinot, B., 1979, in Time and the Earth's Rotation, D. D. McCarthy, J. D. Pilkington (eds), D. Reidel Publishing Company, 7-18. 
Herring, T. A., D. Dong, 1994, J. Geophys. Res., 99, 18051-18071

Lieske, J. H., Lederle, T., Fricke, W., and Morando, B., 1977, Astron. Astrophys., 58, 1-16.

Ma, C., Arias, E. F., Eubanks, M. Fey, A.L., Gontier, A.-M., Jacobs, C. S., Archninal, B. A., Charlot, P., 1998, Astron. J., 116, 516-546.

Mathews, P. M., 1999, Proceedings of the Journées Systèmes de Référence Spatio-Temporels 1998, N. Capitaine (ed.), Observatoire de Paris, 161163.

McCarthy, D. D., 1996, IERS Conventions, IERS Technical Note 21, Observatoire de Paris.

Seidelmann, P. K., 1982, Celest. Mech., 27, 79-106.

T5 Newsletters $(1998,1999,2000)$, http://opdaf1.obspm.fr/danof/T5.html.

Woolard, E. W., 1953, Astr. Pap. Amer. Ephem. Naut. Almanac, XV, I, $1-165$. 